\title{
PRECISION FABRICATION OF HIGH-SPEED MICRO-ROTORS USING DEEP REACTIVE ION ETCHING (DRIE)
}

\author{
Norihisa Miki, Chiang Juay Teo, Linhvu Ho, and Xin Zhang ${ }^{\dagger}$ \\ Gas Turbine Laboratory, Massachusetts Institute of Technology, Cambridge, MA 02139, USA \\ ${ }^{\dagger}$ Department of Manufacturing Engineering and Fraunhofer USA Center for Manufacturing Innovation, Boston \\ University, Boston, MA 02215, USA
}

\begin{abstract}
High-speed silicon micro-rotors require high-precision fabrication via Deep Reactive Ion Etching (DRIE) so as to minimize the rotor imbalance which limits the maximum operational speed. Etch variation of DRIE process culminates in a difference in the blade height and thus rotor imbalance. A Fourier transform of the etch non-uniformity along the rotor circumference revealed the global effect across the wafer and the local etch variation depending on the concentration or proximity of the patterned geometry. The global etch non-uniformity which results in rotor imbalance could be alleviated to $0.25 \%$ across a rotor of $4.2 \mathrm{~mm}$ diameter by optimizing the plasma chamber pressure. The manufacturing processes presented herein are readily applicable to the constructions of other high-speed rotating micro-structures containing intricate geometries and large etched areas.
\end{abstract}

\section{INTRODUCTION}

Rotating micromachinery must spin at high rotating speeds to achieve high power densities, exploiting high-precision bearings and rotors made possible through deep reactive ion etching (DRIE) and multi-stack wafer-level bonding [1], [2]. We have reported DRIE profile control of highly anisotropic, narrow silicon trenches (10-20 $\mu \mathrm{m}$ wide, 300-500 $\mu \mathrm{m}$ deep) [3] and several critical issues involved in the multi-stack silicon direct bonding of the wafers with intricate geometries and large etched areas [4].

Our high-speed microdevice (referred as a "microbearing rig"), consisting of the rotor, bearings, and associated plumbing, is constructed from five DRIE'd silicon wafers precision aligned and fusion bonded into a stack (Figure 1). The rotor is a planar disk with radial turbine blades on one side. It is supported by hydrostatic and/or hydrodynamic gas thrust bearing axially and hydrostatic gas journal bearing radially. Figure 2 depicts the SEM image of the rotor with 22 outer stator blades and 20 inner rotor blades. A typical whirl response curve of a rotor with a certain imbalance is plotted in Figure 3. Whirl amplitude is defined as the distance between the geometric center of the rotor and the journal bearing. Collision occurs when the whirl amplitude exceeds the journal clearance. Below the natural frequency, the rotor spins at its geometric center, which is termed as subcritical operation; above the natural frequency, the rotor rotates about its center of mass, which is termed as supercritical operation [5]. The whirl amplitude during supercritical operation corresponds to the rotor imbalance, which refers to the distance between the geometric center and the center of mass. Rotor imbalance precludes performance of high-speed rotating structures; the maximum peak response near the natural frequency increases with the imbalance, resulting in higher likelihood of rotor collision with the surrounding wall. Higher imbalance also promotes instability, reduces the range of stable operation, and thus limits the maximum operational speeds.
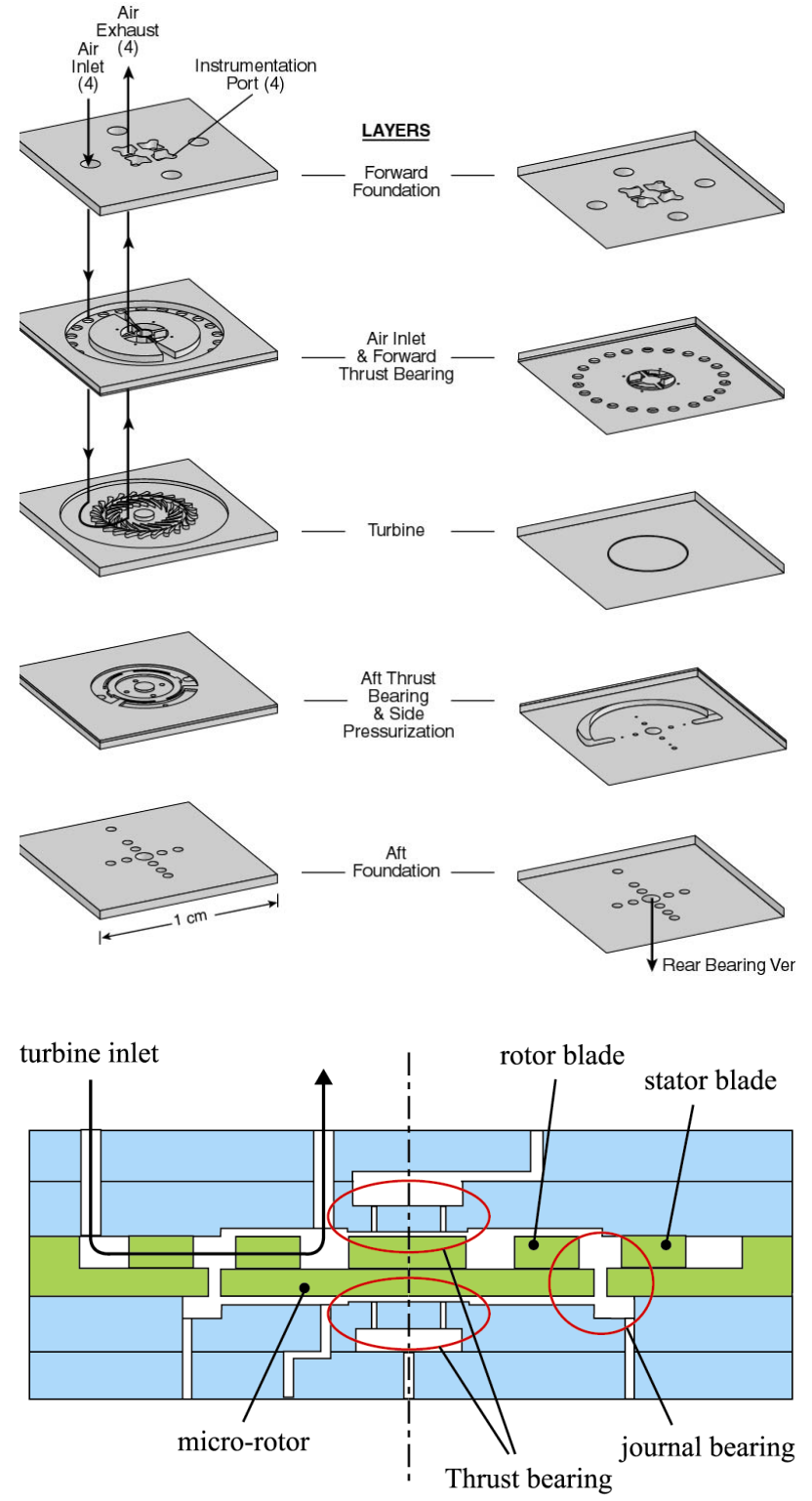

Figure 1. An exploded schematic showing the detailed wafers involved in the microbearing rig. The left column shows the top view, the right column the view from the bottom, and the lower the cross section. The design speed of the $4.2 \mathrm{~mm}$ diameter rotor is 2.4 million RPM. 


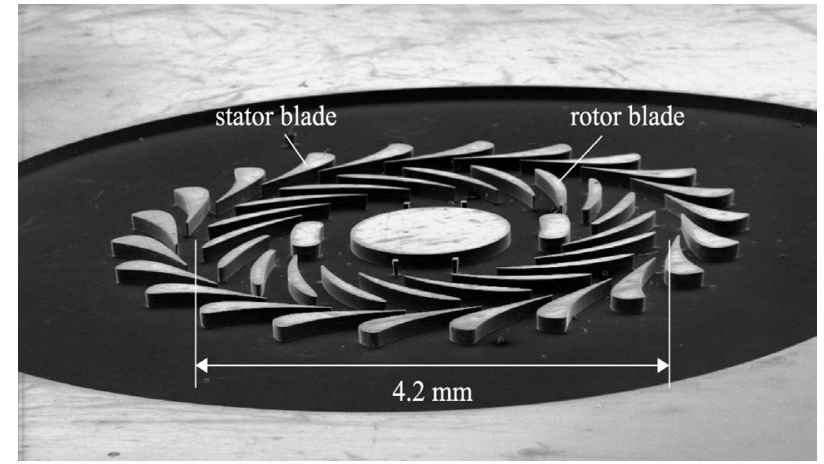

Figure 2. SEM image of a $4.2 \mathrm{~mm}$ diameter by $500 \mu \mathrm{m}$ thick rotor with radial turbine blades on one side (the other side may consist of an electric motor or generator). There are 22 outer stator blades and 20 inner rotor blades fabricated by DRIE. The blade height is $150 \mu \mathrm{m}$.

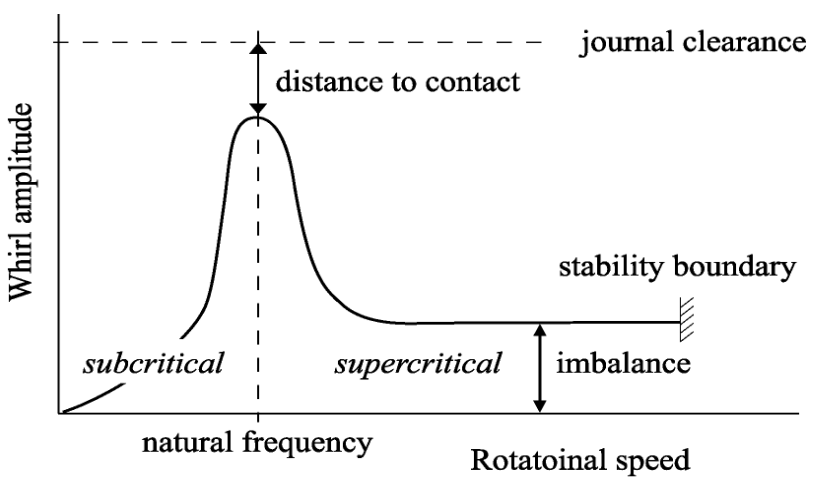

Figure 3. Whirl response of a rotor. The whirl amplitude during supercritical operation represents the rotor imbalance.

One of the major contributors to rotor imbalance is the DRIE etch non-uniformity. The etch variation during the DRIE process culminates in a difference in blade height and thus rotor imbalance. This paper addresses radical DRIE uniformity innovation across $4.2 \mathrm{~mm}$ diameter by $450 \mu \mathrm{m}$ thick rotors; the ultimate goal is to attain a sufficiently low imbalance level of the rotor so as to achieve the design speed of 2.4 million rpm. The DRIE process was performed using a Surface Technology Systems Multiplex ICP.

\section{EXPERIMENTS}

Twelve dies can fit on to one stack of four-inch wafers, as depicted in Figure 4(a). Dies 4, 5, 8, and 9 are identified as center dies, whereas the remaining dies are termed as edge dies. The etch depth is quantified by the maximum depth in the region enclosed by the two adjacent rotor blades and stator blades, such as the area $a_{l}$ surrounded by rotor blades \#1 and \#2 and the closest stator blade as illustrated in Figure 4(b).

Locally, the etch variation depends on the concentration or proximity of the patterned geometry as illustrated in Figure 5. The contour map illustrates the shallower etch depth with the smaller distance to the contiguous features. However, given the symmetric layout of the features, i.e., even numbers of both stator and rotor blades, the local etch variation is identical for diametrically

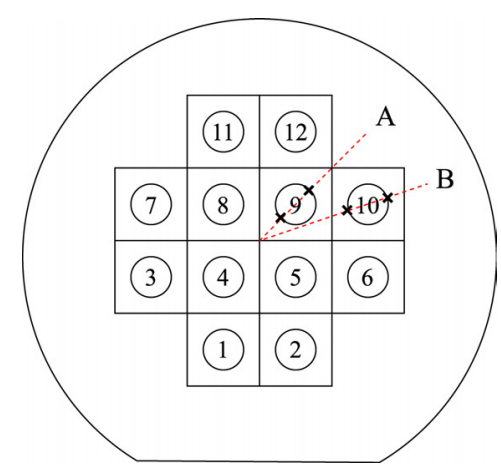

(a)

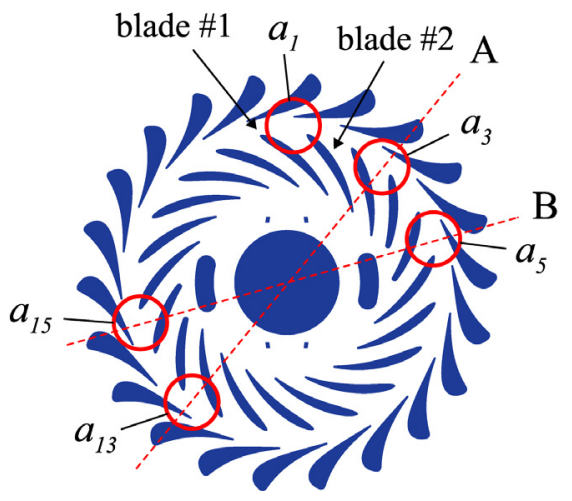

(b)

Figure 4. (a) The location of the dies and (b) the measurement sites.

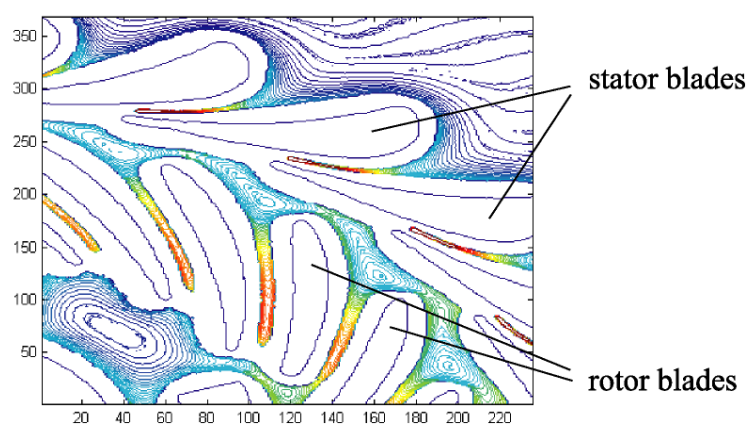

Figure 5. Measured contours showing DRIE etch variation in a micro-rotor.

opposite points on the rotor and thus does not contribute to the rotor imbalance.

Globally, DRIE typically causes the center dies having shallower etches by $\sim 10 \%$ than the edge dies. This is the consequence of a non-uniform etch rate across the wafer. The global etch variation is evaluated from the difference in etch depths corresponding to the two regions which are nearest and furthest from the center of the wafer. For example, the difference in etch depths are measured in regions $a_{3}$ and $a_{13}$ for die \#9 and in regions $a_{5}$ and $a_{15}$ for die \#10. A global etch non-uniformity translates as shown in Figure 6, at the die-level, into a difference in the blade height from one side of the rotor to the other, resulting in the 


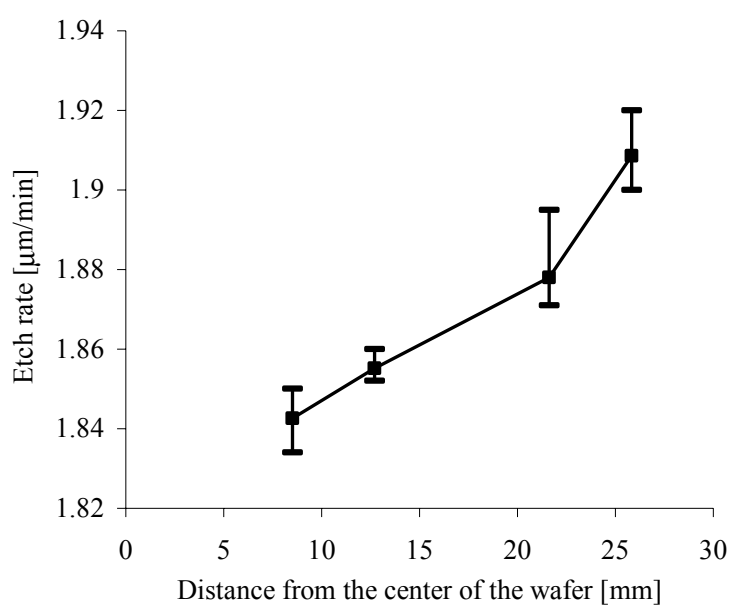

Figure 6. Etch rate variation across the wafer. The etch rates in the regions closest and furthest from the center of the wafer were measured for the 4 center dies and 4 edge dies (die \#1, 6, 7, 12). The left two and the right two represents the global etch nonuniformity across the center dies and the edge dies respectively. The etch rate increases with the distance from the center of the wafer. The etching conditions were: $105 \mathrm{sccm}$ of $S F_{6}(13$ seconds active cycle, 0.5 seconds overlap, $9 \mathrm{~W}$ of electrode power and 750 $\mathrm{W}$ of coil power), $40 \mathrm{sccm}$ of $\mathrm{C}_{4} F_{8}$ (11 seconds active cycle, $6 \mathrm{~W}$ of electrode power and $600 \mathrm{~W}$ of coil power). The APC angle was set at 62 degrees. After 30 minutes of etching, the wafer was rotated through 180 degrees, followed by another 45 minutes of etching, culminating in $150 \mu \mathrm{m}$ high blades.

misalignment between the geometric center and the center of mass, i.e. imbalance.

Figure 7 illustrates the etch depth along the circumference of two different rotors. The etch depth of the area $a_{l}$ corresponds to the datum at the position of 0 degrees. The variation of the etch depth is considered to be the combination of the global and local effects. A Fourier transform was performed to segregate local variations from global contributions (Figure 8). The first harmonic corresponds to the global etch variation, whereas the second harmonic results from the local etch variation due to the symmetric feature arising from the even numbers of rotor and stator blades. The edge die and the center die measured herein involve global etch variations of $5.1 \mu \mathrm{m}$ and $1.4 \mu \mathrm{m}$, respectively.

The imbalance originating from the etch variation was estimated assuming a linear relationship between the global etch non-uniformity and the distance from the center of the wafer. Figure 9 indicates that the imbalance, which is the distance between the geometric center and the center of mass of the rotor, increases almost linearly with the global etch uniformity. For example, a global etch variation of $3 \%$ results in a rotor imbalance of $2.7 \mu \mathrm{m}$. Figure 10 illustrates the whirl response of a rotor with a global etch variation of $3 \%$. The curved line is a fitted line to the typical whirl response curve shown in Figure 3 . In the supercritical region above the natural frequency of $35000 \mathrm{rpm}$, the whirl amplitude representing rotor imbalance was about $3 \mu \mathrm{m}$. This fact indicates that the global etch non-uniformity produced via DRIE process is the dominant factor for rotor imbalance and hence, the performance of high-speed rotors depends on the precision fabrication.

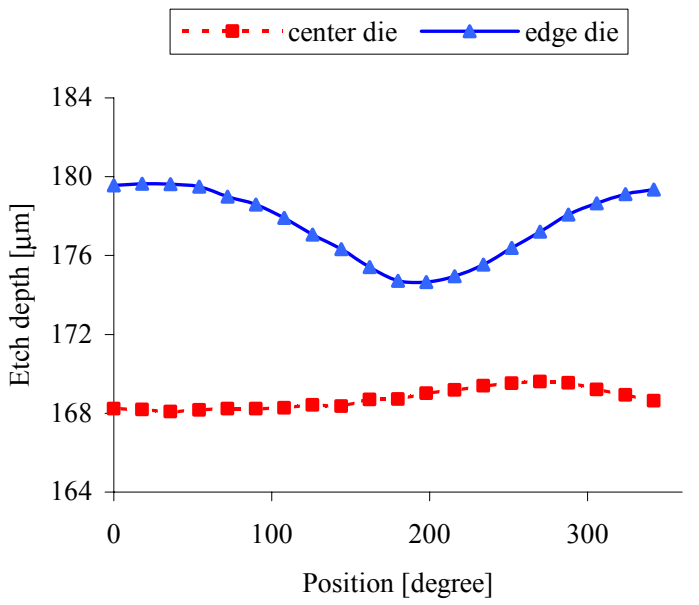

Figure 7. The etch depth along the periphery of the rotor. Plotted along the abscissa is the angular position on the rotor defined in a clockwise manner, with the data point at 0 degrees depicting the maximum etch depth in region $a_{l}$. The etching conditions were identical to those described in the caption of Figure 6.

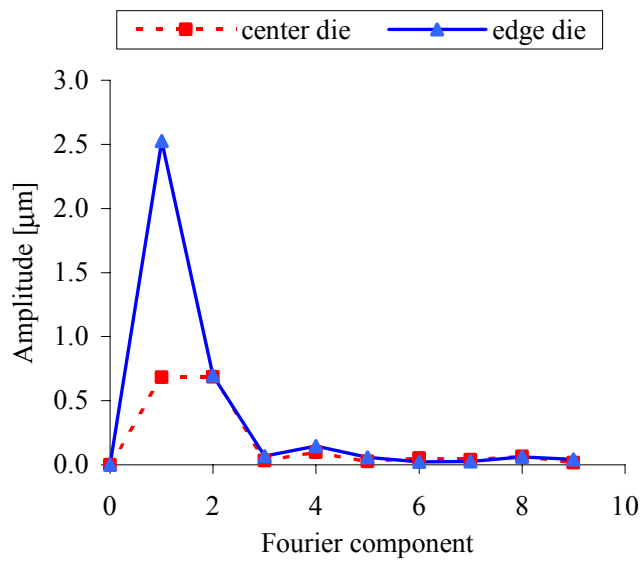

Figure 8. The Fourier spectrum of the etch variation along the periphery of the rotor. The first Fourier component represents the global etch non-uniformity which is a function of the distance from the center of the wafer, while the local etch variation depending on the concentration and proximity of the features appears as the second Fourier component since the number of stator and rotor blades are both even and thus the features are symmetric.

We constructed solutions by regarding the uniformity as having a dependence on (i) the availability of etching species and (ii) the efficient removal of etching byproducts that dissociate and redeposit. As an example, given that the diffusivity of the etching species depends on the etching conditions, we demonstrated approaches to improve etching uniformity by dynamically changing the plasma chamber pressure that is controlled by the automatic pressure control valve (APC) positioning. The plasma chamber pressure increases with the APC angle. Figure 11 depicts the ratio of the etch variation normalized by the etch depth, with respect to the APC angle. The etch non-uniformity was minimized at an APC angle of 55 degrees for both center and edge dies, resulting in the etch variation of $0.25 \%$ and $0.5 \%$ for center dies and edge dies, with corresponding estimated rotor imbalance of 0.2 $\mu \mathrm{m}$ and $0.4 \mu \mathrm{m}$ for center dies and edge dies respectively. 


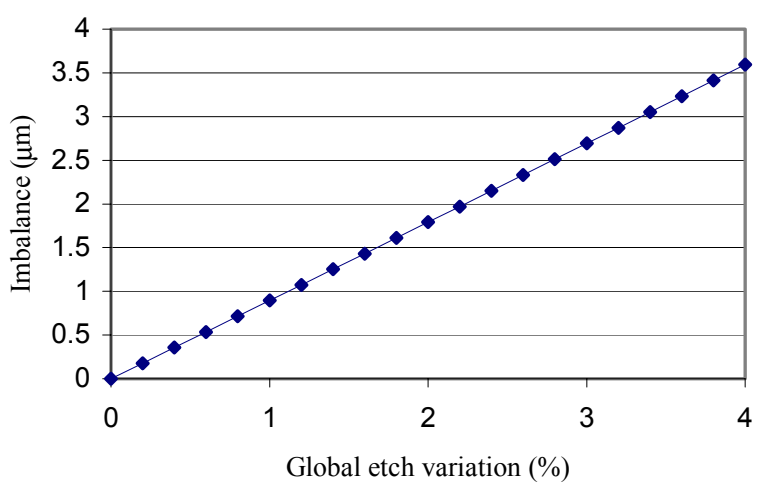

Figure 9. Imbalance caused by the global etch variation. The estimation was conducted with the average blade height of $150 \mu \mathrm{m}$ and the total thickness of $450 \mu \mathrm{m}$.

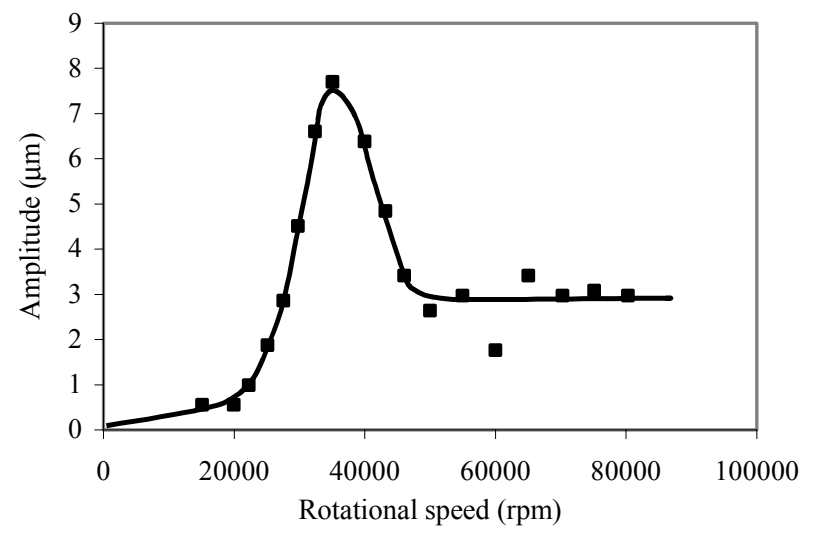

Figure 10. Whirl response of a rotor with a global etch variation of $3 \%$. The whirl amplitude in the supercritical region is about 3 $\mu m$.

\section{CONCLUSIONS}

This paper discusses the etch non-uniformity of DRIE process, which introduces the imbalance on high-speed micro-rotors and limits their performance. The etch variation measured along the periphery of the rotor was the combination of both global and local effects, which could be segregated via Fourier transformation. The imbalance due to the global etch non-uniformity was estimated for the micro-rotor with symmetric features. The comparison to the experimental results revealed the large dependency of the microrotor performance on the precision fabrication. The global etch variation depending on the etching conditions was minimized at an APC angle of 55 degrees. This well-balanced rotor with an estimated imbalance of $0.2 \mu \mathrm{m}$ will be packaged and tested. The manufacturing processes presented herein are readily applicable to the constructions of other high-speed rotating micro-structures and other micro-devices which require precise fabrication and contain intricate geometries and large etched areas.

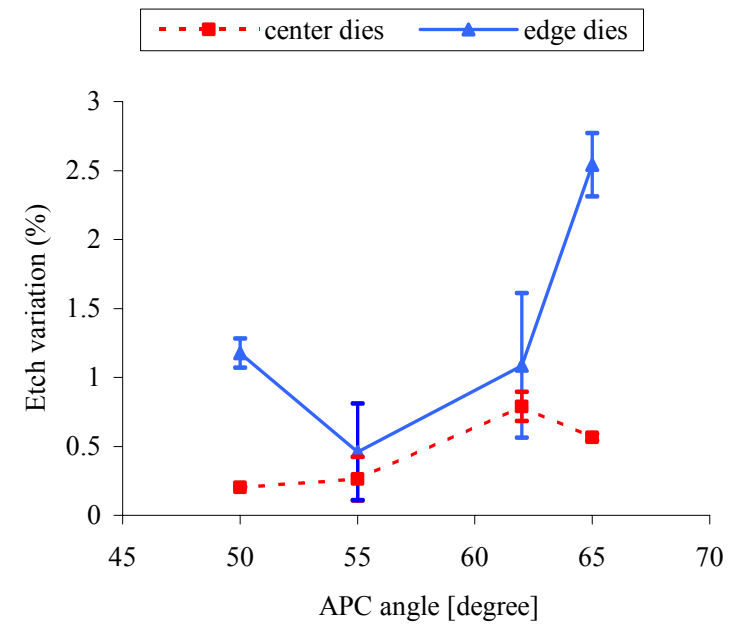

Figure 11. The global etch variation of the center and edge dies with respect to the APC angle. Except the APC angle, the etching conditions were identical to those described in the caption of Figure 6. It can be deduced that an optimal APC angle of 55 degrees culminates in a high yield of well-balanced rotors; these highly precise rotors, with $0.4 \mu \mathrm{m}$ imbalance and $0.25 \% \mathrm{global}$ etch variation, have been repeatedly spun at $1.4 \mathrm{Mrpm}$.

\section{ACKNOWLEDGEMENTS}

This work is supported by the Army Research Office (DAAH04-95-1-0093) under Dr. R. Paur and by DARPA (DAAG55-98-1-0365, DABT63-98-C-0004) under Dr. R. Nowack and Dr. J. McMichael respectively.

\section{REFERENCES}

1. A.H. Epstein and S.D. Senturia, "Macro Power from Micro Machinery," Science, Vol. 276, 1211 (1997).

2. L.G. Frechette, S.A. Jacobson, K.S. Breuer, F.F. Ehrich, R. Ghodssi, R. Khanna, C.W. Wong, X. Zhang, M.A. Schmidt, and A. Epstein, "Demonstration of a Microfabricated HighSpeed Turbine Supported on Gas Bearings," Hilton Head Solid-State Sensors and Actuators Workshop, Hilton Head Island, SC, June (2000), pp. 43-47.

3. A.A. Ayon, X. Zhang, and R. Khanna, "Ultra Deep Anisotropic Silicon Trenches Using Deep Reactive Ion Etching (DRIE)," Hilton Head Solid-State Sensors and Actuators Workshop, Hilton Head Island, SC, June (2000), pp. 339-342.

4. N. Miki, X. Zhang, R. Khanna, A.A. Ayon, D. Ward, and S.M. Spearing, "A Study of Multi-stack Silicon-direct Wafer Bonding for MEMS Manufacturing," The $15^{\text {th }}$ IEEE International Conference on Micro Electro Mechanical Systems, Las Vegas, Nevada, January (2002), pp. 407-410.

5. D. Childs, "Turbomachinery Rotordynamics: phenomena, modeling and analysis," John Wiley, 1993 